\title{
Aporte psicológico do enfermeiro à vítimas de violência doméstica
}

\author{
Psychological support from nurses to victims of domestic violence \\ Apoyo psicológico de enfermeras a víctimas de violencia doméstica
}

Recebido: 29/07/2021 | Revisado: 07/08/2021 | Aceito: 10/08/2021 | Publicado: 14/08/2021

\author{
Paulo Henrique Alves Rosa \\ ORCID: https://orcid.org/0000-0002-5100-2140 \\ Universidade Vale do Rio Verde, Brasil \\ E-mail: paulo.alves@aluno.unincor.edu.br \\ João Paulo Soares Fonseca \\ ORCID: https://orcid.org/0000-0003-4886-1718 \\ Universidade Vale do Rio Verde, Brasil \\ E-mail: joao.fonseca@unincor.edu.br \\ Alessandra Mara Oliveira Dzivielevski \\ ORCID: https://orcid.org/0000-0003-2157-5631 \\ Universidade Vale do Rio Verde, Brasil \\ E-mail: aledzi2@yahoo.com.br \\ Ranile Santos Silva \\ ORCID: https://orcid.org/0000-0002-5844-4224 \\ Universidade Vale do Rio Verde, Brasil \\ E-mail: ranilesantos@yahoo.com.br \\ Daniel Neves Santos \\ ORCID: https://orcid.org/0000-0002-5204-6110 \\ Polícia Civil de São Paulo, Brasil \\ E-mail: ortopediadns@gmail.com \\ Nielly Andrade Carvalho Ribeiro \\ ORCID: https://orcid.org/0000-0002-8399-0657 \\ Universidade Vale do Rio Verde, Brasil \\ E-mail: niellyacr@gmail.com
}

\begin{abstract}
Resumo
A violência contra a mulher é um problema de saúde pública, enraizado por uma questão de gêneroque vislumbra a mulher como objeto de submissão. Levando em conta os altos índices de tentativa de homicídio e homicídio consumado no Brasil e no mundo, onde mediante este cenário torna-se necessário o desenvolvimento de pesquisa sobre o assunto. Com isto, esta pesquisa objetiva investigar a importância do profissional Enfermeiro frente ao atendimento às vítimas de violência doméstica, visto que é necessário conhecimento e preparo do profissional da saúde ao lidar com estas situações, que exigem habilidade emocional e capacidade técnica de modo a contribuir com a possível proteção e cuidados da mulher e da expressão de seus direitos, enquanto cidadã. Os métodos adotados foram alinhados por meio de abordagem quali-quantitativa, colhendo dados teóricos e, em campo para se chegar a resultados precisos e discutir os pontos que necessitam ser trabalhados no Sistema de Saúde para fornecer maior amparo a mulher. Os resultados demonstraram que as participantes em sua maioria, apesar de terem considerado o atendimento recebido na unidade hospitalar adequado, não consideram terem recebido algum tipo de apoio dos profissionais de enfermagem. Com isso conclui-se que existe a necessidade de capacitação desse profissional, sendo necessário intensificar, para que os mesmos forneçam a assistência humanizada adequada, favorecendo o bem-estar da paciente, através do bom aporte psicológico fornecido pelo enfermeiro.
\end{abstract}

Palavras-chave: Violência doméstica; Acolhimento; Assistência de enfermagem.

\begin{abstract}
Violence against women is a public health problem, rooted in a gender issue that sees women as an object of submission. Taking into account the high rates of attempted murder and consummated homicide in Brazil and in the world, where this scenario makes it necessary to develop research on thesubject. With this, this research aims to investigate the importance of the professional Nurse in the care of victims of domestic violence, as knowledge and preparation of the health professional is necessary when dealing with these situations, which require emotional skill and technical capacity in order to contribute with the possible protection and care of women and the expression of their rightsas citizens. The methods adopted were aligned through a quali-quantitative approach, collecting theoretical and field data to arrive at precise results and discuss the points that need to be worked on in the Health System to provide greater support to women. The results showed that most of the participants, despite having considered the care received at the hospital unit adequate, did notconsider that they had received any kind of support from nursing professionals, a fact that demonstrates an increasingly intense need for training for nursing professionals. so that they
\end{abstract}


provide adequate humanized care, favoring the patient's well-being, through the good psychological support provided by the nurse.

Keywords: Domestic violence; Host; Nursing assistance.

\section{Resumen}

La violencia contra la mujer es un problema de salud pública, arraigado en una cuestión de género que ve a la mujer como un objeto de sometimiento. Teniendo en cuenta las altas tasas de tentativa y homicidio consumado en Brasil y en el mundo, donde, a través de este escenario, es necesario desarrollar investigaciones sobre el tema. Con ello, esta investigación tiene como objetivo indagar en la importancia de la Enfermera profesional en la atención de víctimas de violencia intrafamiliar, ya que es necesario el conocimiento y preparación del profesional de la salud ante estas situaciones, las cuales requieren habilidad emocional y capacidad técnica para poder aportar con la posible protección y cuidado de las mujeres y la expresión de sus derechos como ciudadanas. Los métodos adoptados se alinearon a través de un enfoque cuali-cuantitativo, recolectando datos teóricos y, en el campo, para llegar a resultados precisos y discutir los puntos que se deben trabajar en el Sistema de Salud para brindar un mayor apoyo a las mujeres. Los resultados mostraron que la mayoría de los participantes, a pesar de haber considerado adecuada la atención recibida en la unidad hospitalaria, no consideró haber recibido ningún tipo de apoyo por parte de los profesionales de enfermería. Así, se concluye que existe la necesidad de capacitar a estos profesionales, y es necesario intensificar, para que brinden una adecuada atención humanizada, favoreciendo el bienestar del paciente, a través del buen apoyo psicológico brindado por la enfermera.

Palabras clave: Violencia doméstica; Recepción; Cuidado de enfermera.

\section{Introdução}

A violência contra a mulher é uma temática aparentemente simples para quem não compreende a complexidade deste fenômeno, que ocorre num contexto cultural muito forte, em que desde os séculos passados a mulher é vista como propriedade. Contudo, apesar da violência contra a mulher dever-se a muitos fatores diferentes, em quaisquer aspectos, demanda intervenção pública, pois com os altos índices de violência desta natureza no país atualmente, é considerado um grave problema de saúde pública (Souza \& Rezende, 2018).

Inicialmente sabe-se que a violência pode ser expressa de várias formas e possuem níveis de gravidades distintos, que vão desde violência verbal, física, moral, sexual, patrimonial, até a consumação de homicídios. Cabe ressaltar ainda que para mulher existem vários desafios envolvidos para que a mesma se desvincule de um relacionamento abusivo, que vão desde dependência emocional, financeira, medo das consequências, medo de não sentir-se protegida, temor por seus filhos e familiares, falta de segurança pública, entre outros (Leandro, Mata \& Tomé, 2019).

Neste contexto, muitas vezes a mulher procura atendimento de saúde, seja por necessidade de acompanhamento psicológico, seja por lesões corporais, que faz com que o profissional de saúde seja o intermediário entre a vítima e a polícia. Isto porque em casos de violência doméstica, o enfermeiro tem a obrigação de notificar o agravo aos órgãos competentes e auxiliar a paciente a tomar as medidas cabíveis nesta condição.

Com isso, a abordagem do enfermeiro frente ao atendimento a mulheres vítimas de violência doméstica pode constituirse como diferencial para que a mulher sinta-se amparadae representada e denuncie o agressor. Em contrapartida, muitas vezes a mulher busca os atendimentos de saúde e não relatam a veracidade dos fatos, eximindo o agressor de sua culpa, portanto, o atendimento em saúde também pode requerer a detecção destes fatos, para que possa se impulsionar a mulher a sair da sua situação de risco, de forma ética e com o devido cuidado para não pressionar ainda mais, uma mulher que provavelmente já está com o seu emocional fragilizado (Acosta, Gomes, Oliveira, Gomes, \& Fonseca, 2017).

Os altos índices de violência doméstica registrados no Brasil anualmente são alarmantes. Neste contexto e pensando na qualidade do atendimento às mulheres vítimas de violência doméstica, pretende-se responder a seguinte questão: qual o papel do enfermeiro no enfrentamento a violência doméstica?

Sendo assim, a pesquisa tem por objetivo investigar a importância do profissional Enfermeiro frente ao atendimento às vítimas de violência doméstica. 


\section{Metodologia}

Trata-se de pesquisa de natureza quali-quantitativa, de abordagem exploratória, que utiliza dados científicos e experiência pessoal para descrever pontos relevantes sobre mulheres que já foram vítimas de violência doméstica. Segundo Knechtel (2014), pesquisa qualitativa é complexa e está relacionada à essência e descrição do objeto, descrevendo a qualidade ao invés da quantidade, através de raízes filosóficas permitindo a diversidade e flexibilidade. Esse tipo de pesquisa se preocupa em entender ocorrências humanas e processos sociais, buscando uma visão descrita e complexa através de uma análise científica do pesquisador. Sendo assim, o pesquisador tem como critério as motivações, crenças, valores e representações encontradas nas relações sociais (Knechtel, 2014). Já a pesquisa quantitativa, segundo Gil e Vergara (2015), é caracterizada por fases padronizadas, tendo como objetivo final a resposta da hipótese que fora proposta.

A abordagem exploratória se deu através de aplicação de questionário de autoria própria, que foi aplicado às mulheres que foram vítimas de violência doméstica na região do Sul de Minas, na cidade de Três Corações, atendidas nas unidades da Polícia Militarpara registro de ocorrência. Para participar do estudo adotou-se como critério de inclusão: mulheres que sofreram violência doméstica e registraram Boletim de Ocorrência na Unidade na Polícia Militar, e que aceitaram participar da pesquisa por meio do TCLE. Como critério de exclusão: mulheres que sofreram violência doméstica e não fizeram Boletim de Ocorrência e mulheres que desistiram durante a pesquisa.

A amostra proposta contou com a participação de 30 mulheres que foram agredidas no período entre janeiro e junho de 2021, escolhidas de forma aleatória, representando apenas uma parcela do total das mulheres que foram agredidas no mesmo período.

Na fase exploratória da pesquisa, o procedimento ocorreu por meio de aplicação de questionário, destinado a esclarecer as questões pautadas ao longo do referencial teórico. O questionário criado para esta pesquisa é composto de perguntas relativas à ocorrência registrada e sobre o envolvimento da vítima com o agressor, analisado com base nos dados de Boletins de ocorrência efetuados pelaPolícia Militar, após solicitação á vítima, as quais foram procuradas e convidadas a participar da pesquisa, incluindo também questões sobre a percepção das vítimas acerca dos atendimentos que receberam e foi composto por perguntas de múltipla escolha, também foi cedido espaço para declarações sobre a natureza das agressões e dos sentimentos frente à condição de vítima, caso quisessem fazer seus relatos, tanto no que diz respeito ao acolhimento psicológico quanto físico.

O presente estudo respeitou os preceitos estabelecidos pela Resolução ${ }^{\circ}$ 466/12 de dezembro de 2012. Foram respeitados os princípios de anonimato, privacidade e sigilo profissional. Além disso, o participante do estudo teve autonomia para decidir se aceitava ou não participar do estudo.

As entrevistas tiveram início após a aprovação do pré-projeto de pesquisa pelo Comitê de Ética em Pesquisa da Universidade Vale do Rio Verde - UNINCOR. A aplicação do questionário iniciou logo após a aprovação do Comitê de Ética da UninCor, sob parecer (CAAE: 45473521.9.0000.5158). Os questionários foram aplicados pelopróprio autor da pesquisa por meio de visitas domiciliares incluídas na rotina da Polícia Militar, sendo o autor atuante como militar.

Os dados obtidos foram tabulados eletronicamente com auxílio do programa Microsoft EXCEL $® 2010$ e analisados quantitativamente e percentualmente. Já análise qualitativa foirealizada pelo próprio autor da pesquisa com as informações do questionário.

\section{Resultados e Discussão}

Responderam a pesquisa trinta participantes, que relataram informações acerca da condição de vítimas de violência doméstica. Nenhuma entrou nos critérios de exclusão. A partir da análise dos dados inciais, nota-se que todas as vítimas são mulheres. Quanto a faixa etária, nota-se que a ocorrência de violência doméstica não ocorre em uma faixa etária específica, pois ainda que a prevalênciana pesquisa seja entre as mulheres de 20 a 30 anos, mulheres de 41 anos ou mais também são 
vítimas.

Ainda acerca dos dados sociodemográficos, percebe-se que a maioria das participantesdeclararam serem "do lar", sendo 70\% delas, onde evidencia-se que não há existência de renda própria, causando muitas vezes a dependência financeira do cônjugue ou agressor, informaçãoque será tratada ao longo dos resultados. Também há estudante (3,33\%) e outras $10 \%$ possui um regime informal de trabalho, nos quais realizam atividades que não proporcionam renda fixa, entre as fontes de renda citada neste caso estão: lavadeira, catadora de recicláveis e artesã, ou seja, são fontes de rendas sazonais e que também podem vir a limitar as mulheres quanto as suas necessidades e tomadas de decisões. As mulheres com registro na CTPS - Carteira de Trabalho e Previdência Social somam apenas 13,33\% dos resultados da pesquisa.

Inciando pelo causa do registro de ocorrência, evidenciou -se múltiplos motivos apontados pelas participantes, sendo a agressão consumada descrita por todas as entrevistadas, bem como outras atitudes violentas, como tentativas de agressão, ameaças verbais e até mesmo tentativa de homicídio contra as participantes ou pessoas pertencentes ao seu círculo familiar.

Sob este cenário, é importante dizer que desde ameaça, até a agressão consumada, são consideradas formas de violência e são relatadas como consequências posteriores a violência, as tentativas de suicídio, pressão arterial descontrolada, dificuldades na vida sexual e profissional (Netto, Moura, Queiroz, Tyrrell, \& Bravo, 2014).

Com isso, cabe evidenciar a importância da implantação da Lei Maria da Penha $n^{\circ} 11.340 / 2006$, a qual nasceu da gravidade da violência sofrida pela mulher que deu o nome a lei. O objetivo desta lei é abrangente no sentido de amparo a vítima, a qual também medidas de urgência que são visualizadas no mecanismo impeditivo para que violência se repita, maioria dos mecanismos é de conhecimento popular, que voltam se tanto a vítima quanto ao agressor. Algumas por sua vez obrigam o agressor a ficarem afastados da vítima através de medidas protetivas, devem ser devidamente estudadas, pois nem sempre oferecem um bom resultado e tem sido tema de constante debate no Brasil (Agência Brasil, 2019).

Também podem-se citar as consequências físicas persistente referentes às lesões, como hematomas escoriações, luxações, fraturas, partos prematuros, mutilações, queimaduras, entre outras condições relacionadas a violência sexual, como abortos, doenças sexualmente transmissíveis,estresse pós-traumático e em casos extremos ocorre a consolidação da violência na morte da vítima (Martins, Batista, \& Divino, 2017).

Em seguida foram questionadas acerca do grau de parentesco com o autor das agressões, sendo 96,67\% trata-se de relações amorosas atuais ou passadas, sendo que somente 3,33\% é vítima de violência doméstica, por parte de seu filho.

Foi perguntado também se as participantes, já haviam registrado ocorrências anteriormente, 50\% relatou que não, e as demais relataram que sim, sendo $20 \%$ pelo menos uma vez e 30\% mais de uma vez, demonstrando a recorrência da ação agressiva em pelo menos metade dos casos. Após isso, foi questionado se as participantes ainda mantinham convivência ou relacionamento com os agressores, neste contexto, a maioria representada por 53,3\% responderam que sim, e 46,7\% que não.

Ocorre que as mulheres naturalizam suas condições, por não verem opções paratomarem decisões, ou por acreditarem que as condutas violentas dos seus companheiros devem ser aceitas, pois são de algum modo interiormente justificada, seja pela posição de dominação masculina na relação do casal, seja devido a sentimentos de amor, ou a atribuírema violência exclusivamente ao uso de substâncias, por exemplo (Guimarães et al., 2018).

Neste contexto é importante dizer que diversos fatores podem levar a permanência no relacionamento ou a não ruptura do convívio com os agressores, motivos que foram também questionados, e que foram descritos como dependência emocional e segundo as mesmas, os sentimentos de amor e apego pelo parceiro ainda são fortes mesmo com as agressões, seguido também do medo, dependência financeira, e os filhos também são declarados como justificativa.

A dependência emocional como critério pra permanecer na relação tem sido muito debatida na literatura, pois naturalmente se espera da pessoa agredida, que ela deixe de possuir qualquer sentimento bom de afeto pela pessoa. No entanto, as vítimas de violência doméstica estão normalmente relacionadas a um jogo emocional, por parte do agressor que gera 
esperanças e expectativas na mulher sobre melhoras no seu comportamento. Sendo o agressor a pessoa que realiza as promessas, e a mulher com a mente fragilizada e com a dependência emocional não consegue sair da situação, mas não se trata de sentimento de amor, mas sim de uma dependência, a qual passa a ser inconscientemente controlada, tendo medo de perder o afeto do seu parceiro, aceitando então ser subjugada, com sentimentos de incapacidade sobre si mesmo (Silva \& Silva, 2020; Vásquez \& Rojas, 2020).

Os tipos de agressão sofrida pelas mulheres estão no Gráfico 1, a seguir:

Gráfico 1 - Tipos de agressão sofrida pelas vítimas.

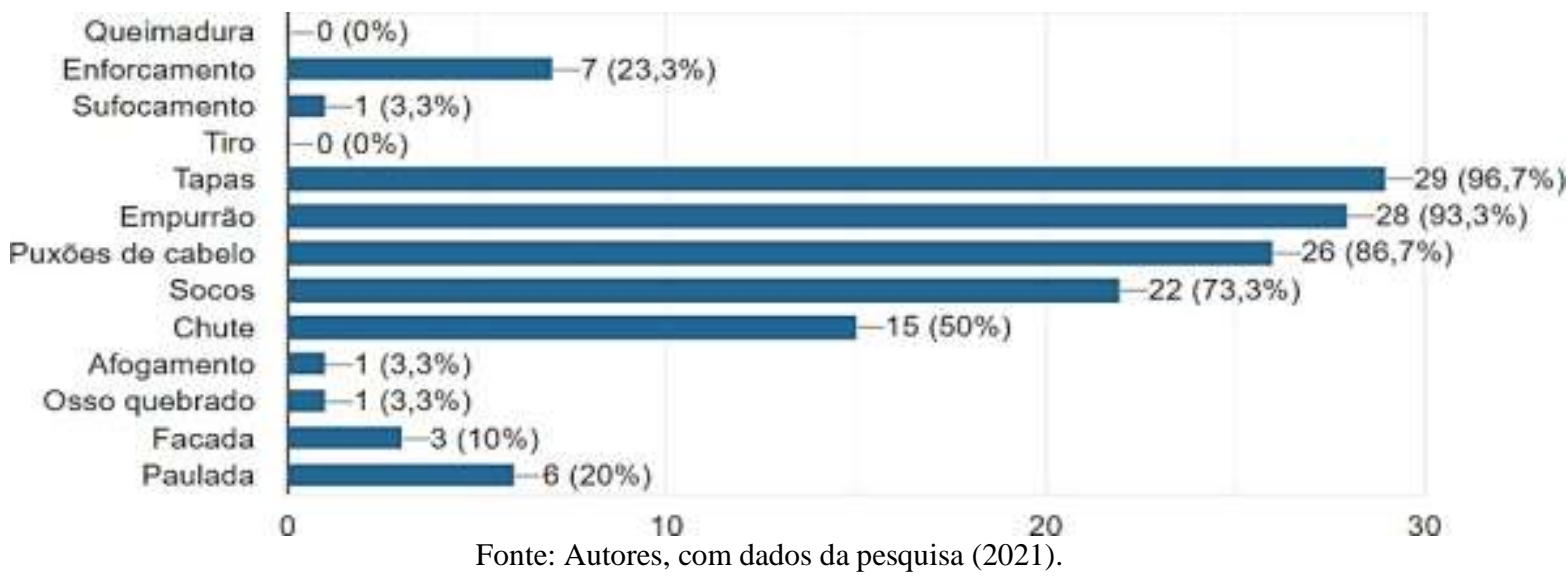

No gráfico, é possível perceber as formas de agressão mais mencionadas são tapas, empurrões, puxões de cabelo, socos e chutes, sendo ainda outras formas mais graves citadas como facadas, afogamento, enforcamento e paulada. A gravidade das agressões e a recorrência, é algo muito comum nas estatísticas brasileiros. Assim como mencionado anteriormente, o medo é uma das causas que fazem com que as mulheres se mantenham no relacionamento, mas as dependências também, as quais precisam ser tratadas sob os panoramas sociais necessários.

Além da gravidade das lesões que pode comprometer a saúde física, entre os agravos de saúde mental provenientes das formas de agressões mencionadas sepodem citar, de acordo com Martins et al. (2017) cefaleias persistentes, dores musculares, transtornos de ansiedade e outros agravos mentais, como depressão, estresse, sensação de cansaço extremo, emagrecimento, distúrbios gastrointestinais, distúrbios de sonoe alimentação como obesidade, isolamento social, sentimento de culpa, insegurança, autoestima baixa, aumento do uso de álcool e drogas, síndrome do pânico, acidente vascular cerebral, paralisia facial, entre outros.

Sobre possuir filhos com o autor das agressões $60 \%$ relatou que sim, contra $40 \%$ que relataram que não. Esta pergunta encerrou a parte um do questionário, ficando a próximaetapa destinada a percepção do atendimento que as vítimas receberam nas esferas policiais e de saúde. Sendo que acerca dos terem recebido atendimento adequado da polícia Militar de Minas Gerais, $96,7 \%$ responderam que sim.

É importante ressaltar que além do sentimento de insegurança, a mulher também sofre com baixa autoestima e vergonha, pois muitas vezes, não existe uma compreensão por parteda sociedade sobre suas subjetividades, pois o fato de não colocar um ponto final na violência, às vezes resulta também em violência institucional, de modo que a mulher nãovolte a buscar ajuda nas instituições da qual ela não se sentiu acolhida e o ciclo de violênciase mantém, porém a compreensão e acolhimento são necessários, pois a busca por ajuda pode ser um caminho para que a mulher não seja vítimas de feminicídio, tornando a situação ainda mais grave para a sociedade como um todo (Goes, 2019).

Quanto a terem recebido adequado no atendimento hospitalar, fica evidente que a maioria considera ter recebido 
atendimento adequado na unidade hospitalar, sendo 83,3\% das participantes, mas ainda assim, 16,7\% consideram que não, o que representa ainda um percentual muito elevado, pois a assistência a saúde integral e adequada é dever do estado e ocorre através do empenho de profissionais comprometidos e dedicados.

Referente ao preparo do Sistema de Saúde em atender esta demanda, de acordo com Minayo, Souza, Silva, e Assis (2018), o SUS cumpre um papel de prestar atendimento de uma forma objetiva, respeitando os valores e singularidades das mulheres, pois segundo ele é importante entendero contexto sociocultural que a mulher vive e respeitá-la sem qualquer discriminação.

Para este preparo pudesse ser efetivo, medidas deveriam ser tomadas para que estas mulheres fossem devidamente acolhidas nos sistemas de Saúde. A Secretaria Nacional de Saúde publicou a Política Nacional de Atenção Integral à Saúde da Mulher, que tem como diretrizes a humanização e qualidade nos atendimentos às mulheres nos serviços de saúde. Ademais, é necessária a orientação e capacitação dos profissionais em relação à assistência das mulheres, observando as perspectivas de gênero e dando ênfase também as etnias em consonância aos seus direitos, considerando o cenário estatístico de violência contra a mulher no Brasil (Minayo, 2018).

Assim, na sequência, foi questionado aos participantes, se os mesmos consideraram receber algum tipo de apoio da equipe de enfermagem, quanto aos atendimentos que receberam após as agressões, onde pode-se dizer que os resultados foram supreendentemente negativos, tendo em vista que a grande maioria das participantes, o que totaliza 93,3\% considera não ter recebido nenhum tipo de apoio da equipe de enfermagem, quando buscou atendimento sobre as agressões sofridas. Estes números representam uma insatisfação expressiva por parte das vítimas, mas também muitas podem acreditar não ser responsabilidade da equipe de enfermagem fornecer apoio e acolhimento.

Isto porque muitas vezes, estas mulheres não têm conhecimento de que os serviços de saúde têm orientação e capacitação para proporcionar acolhimento a esta demanda da sociedade, e não revelam aos profissionais a verdadeira causa da procura pelos serviços, apenas relatam os sentimentos normalmente descritos como angústia, depressão e o nervosismo. Os profissionais, por sua vez fazem a medicação para apaziguar os sintomas e acabam não podendo prestar o suporte que deveriam devido ao desconhecimento das causas dos determinados sintomas, com isso o problema torna-se cada vez mais difícil de ser solucionado (Souza \& Rezende, 2018).

No entanto, é indiscutível pela literatura que o cuidado humanizado na enfermagem proporciona melhores resultados para o paciente, quando equiparados a um cuidado estritamente técnico. Pois, tento em vista que a maioria também considerou ter recebido tratamento adequado nas unidades de saúde, é possível relacionar estas considerações de quea parte técnica pode ter sido bem gerida, diferente do cuidado e acolhimento humanizado neste sentido (Acosta et al., 2017).

O enfermeiro tem ainda, um papel essencial no que diz respeito também ao enfrentamento da violência a nível regional, pois quando constatada a agressão, é necessário que o mesmo notifique seus superiores que tem por obrigação legal levar esta informação aos órgãos competentes. No Brasil, esse registro é feito pela SINAN (Sistema de Notificação de Agravos de Notificação) (Cevs, 2020). Cabe salientar ainda que não precisa que a agressão seja efetivamente relatada pela vítima, sendo os casos suspeitos também notificáveis, como obrigatoriedade, imposto pela Portaria nº 204 de 17/02/2016, que determina todos os agravos de saúde que devem ser notificados (Brasil, 2016).

Outro fato que muitas vezes impede o profissional da saúde em relatar a notificação é o temor por represálias, o constrangimento emquestionar detalhes da violência e também a naturalização da mesma, quando, infelizmente osprofissionais banalizam como se fosse um fato corriqueiro do cotidiano, conforme argumenta Kind et al. (2013). Tratando-se singularmente o profissional de saúde, cabe enfatizar que o preparo que o enfermeiro tem ocorre no meio acadêmico e ainda que sejam implantadas políticas Públicas, não será tão eficaz se o profissional não tiver a bagagem de conhecimento que necessita para lidar com esta situação (Narvaz \& Koller, 2007). 
Questionadas se sentiram-se seguras dentro das unidades hospitalares em que foram atendidas, onde, $66 \%$ responderam que sim e 33,3\% que não, demonstrando entre estas 33,3\% um medo exacerbado, pois mesmo num ambiente cercado de pessoas e monitoramento, as mesmas expressam receios por partes dos seus parceiros ou ex-parceiros agressores.

Ainda é importante dizer que no caso desta pesquisa a busca por atendimento de saúdese deu devido a lesões físicas, no entanto, Souza e Rezende (2018), relatam que as mulheres podem também recorrer aos serviços de saúde para tratar problemas em suma maioria, denatureza emocional, que se manifestam frequentemente em palpitações, ansiedade e problemas digestivos.

Outro ponto a ser discutido é a violência doméstica que tem ocorrido durante a pandemia, em que o isolamento social, muitas vezes mantém as mulheres convivendo mais tempero em cárcer com seus agressores, onde as mulheres muitas vezes acham meios não convencionais para delatar sua condição, como ligar para polícia e pedir pizza, como forma de ter acesso a mesma e outras formas de manifestação já divulgadas pela mídia que são usadas como escape por muitas mulheres em situações de violência (Isto É, 2021).

Mas, sabe-se ainda que apesar de o isolamento social corroborar de certa forma com a repressão de mulheres vítimas de violência doméstica, estima-se que ainda antes da pandemia, um quantitativo inferior a 40\% das mulheres vítimas de violência doméstica buscavam ajuda, sendo que menos que $10 \%$ dessas vítimas buscavam à polícia, tendo em vistas que muitas recorrem aos familiares e não consumam um registro de ocorrência (Oliveira \& Onuma, 2020).

Considerando a necessidade de enfrentamento á violência doméstica, campanhas são realizadas ao redor do país para prestar suporte e prevenir a violência contra as mulheres, principalmente durante a pandemia, como ocorreu em Minhas através do lançamento da Campanha: "Distanciamento Social sem Violência Doméstica", que tem intuito preventivo, onde se divulgam contatos para se fazer denúncias, são realuzados encontros para discutir estratégias e métodos para o enfrentamento da violência doméstica em tempos atuais, incluindo a adesão de políticas públicas de amparo (Agência Minas, 2021).

Com isso, os resultados levam a reflexão sobre a necessidade constante de amparo psíquico e emocional para estas mulheres vítimas de violência doméstica e o enfermeiro é uma parte importante, pois assume papel de intermediador e de comunicador e toda assistência humanizada possível será importante para a vítima se sentir respeitada e acolhida.

\section{Conclusão}

A partir da realização desta pesquisa, nota-se que a violência doméstica implica em impactos graves sobre a vida das vítimas, colocando-as em posição de risco constante de morte, gerando também lesões físicas, mas também muitos impactos de origem emocional, que podem requerer a busca por cuidados de saúde.

Também há de se fomentar as consequências a nível social proveniente deste tipo de violência, pois o tanto o desenvolvimento de doenças quanto as potenciais perdas nos aspectos pessoais, afetivos, sociais e econômicos, resultam claramente em prejuízoeconômico e social tanto para a saúde e qualidade de vida da mulher como para a sociedade. No entanto, o que se enfatiza nesta pesquisa é o aporte psicológico que o enfermeiro pode fornecer a vítima, com base nas necessidades psicológicas e emocionais, ficou evidente nesta pesquisa que o enfermeiro assume um papel fundamental e que o cuidado humanizado eintegral é indispensável na busca por resultados positivos no atendimento a vítima. É fato também que alguns fatores externos podem dificultar a assistência.

Sendo assim, há necessidade de capacitação constante para que o enfermeiro consiga dar o aporte psicológico necessário a vítima, compreendendo-a em suas subjetividades e direcionando na busca de cuidados especializados, para isso, a sistematização de assistência de enfermagem e a ética, devem ser aplicadas.

Sugere-se, como trabalhos futuros, conhecer as repercussões psíquicas na vida da mulher que sofre de violência doméstica. Ademais, é necessário a elaboração de pesquisas que avaliem a eficácia de treinamentos específicos direcionados aos 
enfermeiros para atendimento de vítimas de violência doméstica.

\section{Referências}

Acosta, D. F., Gomes, V. L. O., Oliveira, D. C., Gomes, G. C., \& Fonseca, A. D. (2017). Aspectos éticos e legais no cuidado de enfermagem àsvítimas de violência doméstica. Texto \& Contexto-Enfermagem; 26(3), e6770015.

Agência Minas. (2021). Campanha do Governo previne a violência contra as mulheres durante pandemia. http://www.agenciaminas.mg.gov.br/sala-deimprensa/campanha-do-governo-previne-a-violencia-contra-as-mulheres-durante-pandemia

Brasil. Ministério da Saúde. (2016). Portaria nº 204 de 17 de fevereiro de 2016.

Agência Brasil. (2019). Senado aprova mudanças para endurecer Lei Maria da Penha. https://www.correiobraziliense.com.br/app/noticia/polit ica/2019/ 08/07 /int erna_politica,775985/lei-maria-da-penha-mais-rigida.shtml

CEVS. Centro Estadual de Vigilância em Saúde. Tipologia da violência. Recuperado em 22 agosto 2020 https://www.cevs.rs.gov.br/tipologia-daviolencia

Gil, A. C. \& Vergara, S. C. (2015). Tipo de pesquisa. Universidade Federal de Pelotas, Rio Grande do Sul, RS, Brasil.

Goes, E. D. A. (2019). A vergonha social e o medo: obstáculos para a superaçãoda violência doméstica contra a mulher. Brazilian Journal of Development; 5(11): 23627-45.

Guimarães, R. C. S., Soares, M. C. S., Santos, R. C., Moura, J. P., Freire, T. V. V., \& Dias, M. D. (2018). Impacto na autoestima de mulheres emsituação de violência doméstica atendidas em Campina Grande, Brasil. Revista Cuidarte; 9(1): 1988-97.

Isto É. (2021). Mulher liga para 190 pedindo pizza e policial entende pedido de socorro. https://istoe.com.br/mulher-liga-para-190-pedindo-pizza-e-policialentende-pedido-de-socorro/

Knechtel, M. R. (2014). Metodologia da pesquisa em educação: uma abordagem teórico-prática dialogada. Intersaberes.

Kind, L., Orsini, M. L. P., Nepomuceno, V., Gonçalves, L., Souza, G. A., \& Ferreira, M. F. F. (2013). Subnotificação e (in)visibilidade da violência contra mulheres na atenção primária à saúde. Cad. Saúde Pública; 29(9): 1805-15.

Leandro, G. V., Mata, A. L. S., \& Tomé, S. F. (2019). Violência Doméstica E Feminicídio: Uma Análise Acerca Das Consequências De Um Relacionamento Abusivo. Recuperado em 22 agosto 2020 http://publicacoesacademicas.unicatolicaquixada.edu.br/index.php/eedic/article/view/3832/3301

Martins, M. C. V., Batista, A. C., \& Divino, A. E. A. (2018). A Sistematização da Assistência de Enfermagem no atendimento a mulheres vítimas de violência. Caderno de Graduação-Ciências Biológicas e da Saúde; 4(3): 113-22.

Minayo, M. C. S., Souza, E. R., Silva, M. M. A., \& Assis, S. G. (2018). Institucionalização do tema da violência no SUS: avanços e desafios. Ciência \& Saúde Coletiva; 23(6): 2007-16.

Narvaz, M. G., \& Koller, S. H. B. (2017). A marginalização dos estudos feministas e de gênero na psicologia acadêmica contemporânea. Psico; 38(3), 216-223.

Netto, L. A., Moura, M. A. V., Queiroz, A. B. A., Tyrrell, M. A. R., \& Bravo, M. M. P. (2014). Violência contra a mulher e suas consequências. Acta Paul Enfermagem; 27(5): 458-63.

Oliveira, A. L. \& Onuma, F. (2020). "Paradoxo da Pandemia” no registro de casos de Violência Doméstica contra Mulheres nas quatro cidades mais populosas do Sul de Minas Gerais. https:/www.unifal-mg.edu.br/portal/2020/06/05/o-paradoxo-da-pandemia-no-registro-de-casos-de-violencia- domesticacontra-mulheres-nas-quatro-cidades-mais-populosas-do-sul-de-minas- gerais/

Silva, D. \& Silva, R. L. F. C. (2020). Violência contra as mulheres nosrelacionamentos conjugais e a dependência emocional: fator que influencia a permanência na relação. Humanidades e Tecnologia (Finom); 20(1): 328-40.

Souza, T. M. C. \& Rezende, F. F. (2018). Violência contramulher: concepções e práticas de profissionais de serviços públicos. Est. Inter. Psicol.; 9(2): $21-38$

Vásquez, H. R. B. \& Rojas, E. P. P. (2020). Dependência emocional como fator de risco para a violência familiar, um problema de saúde pública. Revista Peruana de Ciências da Saúde; 2(4): e226. 